\title{
Orthostatic hypotension in older adults: the role of medications
}

\author{
Giulia Rivasi, Andrea Ungar \\ Hypertension Referral Centre, Department of Geriatrics and Geriatric Intensive Care Unit, Careggi Hospital and \\ University of Florence, Italy
}

\begin{abstract}
Orthostatic hypotension $(\mathrm{OH})$ is defined as an abnormal blood pressure reduction when standing and is frequently diagnosed in older adults. Pharmacological therapy is one the main causes of orthostatic blood pressure impairment, leading to iatrogenic $\mathrm{OH}$. Indeed, several medications may induce hypotensive effects and influence the blood pressure response to orthostatism. Hypotensive medications may also overlap with other determinants of $\mathrm{OH}$, thus increasing the burden of symptoms and the risk of complications. Potentially hypotensive medications include both cardiovascular and psychoactive drugs, which are frequently prescribed in older patients. According to the available evidence, the antihypertensive treatment "per se" does not seem to predispose to $\mathrm{OH}$, even if a higher risk is associated with polypharmacy and drug classes such as with diuretics and vasodilators. As concerns psychoactive medications, $\mathrm{OH}$ is a well-known adverse effect of tricyclic antidepressants, trazodone and antipsychotics.
\end{abstract}

Correspondence: Giulia Rivasi, Geriatric Intensive Care Medicine, Hypertension Centre, Syncope Unit, University of Florence and Azienda Ospedaliero-Universitaria Careggi, Largo Brambilla 3, 50139, Florence, Italy.

E-mail: giulia.rivasi@gmail.com

Authors' contributions: GR, AU, conception and design, interpretation of data, drafting the article or revising it critically for important intellectual content and final approval of the version to be published. Both authors agreed to be accountable for all aspects of the work.

Conflict of interest: none declared.

Ethics approval and informed consent: No ethical committee approval was required for this study, because this article does not contain any studies with human participants or animals.

Key words: orthostatic hypotension; blood pressure; antihypertensives; psychoactive drugs.

Received for publication: 21 February 2020.

Accepted for publication: 22 April 2020.

CCopyright: the Author(s), 2020

Licensee PAGEPress, Italy

Monaldi Archives for Chest Disease 2020; 90:1254

doi: 10.4081/monaldi.2020.1254

This article is distributed under the terms of the Creative Commons Attribution Noncommercial License (by-nc 4.0) which permits any noncommercial use, distribution, and reproduction in any medium, provided the original author(s) and source are credited.
The knowledge of hemodynamic consequences of drug therapy may be helpful to improve $\mathrm{OH}$ treatment. A medication review is advisable in all patients presenting with $\mathrm{OH}$, particularly at advanced age, aiming at optimizing medical treatment with a view to minimize the risk of iatrogenic $\mathrm{OH}$.

\section{Introduction}

Orthostatic hypotension $(\mathrm{OH})$ is defined as a fall of at least 20 $\mathrm{mmHg}$ in systolic blood pressure and/or $10 \mathrm{mmHg}$ in diastolic blood pressure within 3 minutes of standing [1]. It is highly prevalent in older adults [2,3] and is known to be associated with both short- and long-term adverse outcomes, including falls, cardiovascular events, cognitive impairment and mortality [4-6].

$\mathrm{OH}$ is usually classified according to the presence of a neurodegenerative etiopathogenesis, thus identifying neurogenic and non-neurogenic forms. The latter may derive from reduced plasma volume, deconditioning and exaggerated venous pooling, but also include iatrogenic or drug-related $\mathrm{OH}$. Indeed, several medications may have hypotensive effects and influence the orthostatic blood pressure response. Iatrogenic $\mathrm{OH}$ represent the most common form of non-neurogenic $\mathrm{OH}$, particularly in older patients [4]. Moreover, it may coexist with other causes of $\mathrm{OH}$, thus worsening the patients' symptoms and the risk of complications.

Data from the French pharmacovigilance service suggests that iatrogenic $\mathrm{OH}$ represents the $1.3 \%$ of drug adverse reactions [7]. It is more common in people presenting with several risk factors for $\mathrm{OH}$, such as Parkinson disease, dehydration and diabetes [7], or taking more than one predisposing medications [8]. Additionally, the risk of iatrogenic $\mathrm{OH}$ increases with advancing age. Indeed, older adults frequently present a high risk profile for $\mathrm{OH}$, due to comorbidities, polypharmacy and deconditioning. Moreover, age-related pharmacokinetic changes usually occur, that may alter the bioavailability and distribution of medications [9].

Drug therapy may induce $\mathrm{OH}$ through different mechanisms. Some drug classes interfere with reflex responses that usually counteract a blood pressure fall when standing, e.g., sympatheticmediated vasoconstriction and increased heart rate response and inotropism. Additionally, some medications may increase venous pooling (e.g., vasodilators) and/or induce volume depletion (e.g., diuretics), thus predisposing to $\mathrm{OH}$.

The association between $\mathrm{OH}$ and medications has been widely investigated in the literature, with heterogeneous and partly inconsistent results. However, on the basis of the available evidence, some "high risk" drug classes can be identified.

Anti-hypertensive medications are usually considered to be a predisposing factor for $\mathrm{OH}$. Yet, some evidence suggests that antihypertensive treatment may not increase the risk of an orthostatic 
blood pressure fall, but rather have a protective effect [10-12]. Indeed, uncontrolled hypertension has been demonstrated to favor $\mathrm{OH}$, probably due to a natriuretic effect $[2,12,13]$. Consistently, blood pressure control with antihypertensive treatment reduced the prevalence of $\mathrm{OH}$ in a previous study by Masuo et al., regardless of the drug class used [14]. Therefore, antihypertensive treatment "per se" does not seem to increase the patient's predisposition to orthostatic blood pressure fall. However, the risk of $\mathrm{OH}$ is known to increase with increasing number of antihypertensive medications, particularly when higher than three $[2,8,13]$.

Among antihypertensive medications, some drug classes show a stronger association with $\mathrm{OH}$. These include diuretics and vasodilating agents such as nitrates and $\alpha$-adrenergic receptor antagonists, that reduce circulating volume, venous return and peripheral vascular resistance [8,15-16]. Additionally, beta-blockers may impair the orthostatic blood pressure response, due to their negative inotropic and chronotropic effects $[12,17,18]$. Among beta-blockers, hypotensive effects are expected to be more relevant for mixed alpha- and beta-receptors blockers, e.g. carvedilol, as compared with highly selective molecules, e.g. bisoprolol [19]. Conversely, ACEinhibitors and angiotensin receptor antagonists have a higher tolerability and a low risk of $\mathrm{OH}$, with some data suggesting a protective effect against the orthostatic blood pressure fall $[12,17,20]$. Yet, in older adults with dementia an association with syncope due to $\mathrm{OH}$ has been reported for ACE-inhibitors, when prescribed in a combination with nitrates or diuretics [21]. Therefore, drug interactions may further increase the risk of iatrogenic $\mathrm{OH}$, particularly in more vulnerable population, e.g. people with dementia.

In addition to cardiovascular medications, some psychoactive drugs may likewise induce hemodynamic effects and influence the blood pressure response to orthostatism. Indeed, $\mathrm{OH}$ is the most commonly reported cardiovascular side effect of psychoactive therapies. $\mathrm{OH}$ is reported in $10-50 \%$ of patients taking tricyclic antidepressants, due to the blockade of alpha-adrenergic receptors $[16,20]$. A similar mechanism is described for trazodone [8] and antipsychotics $[22,23]$, the latter being more prone to induce $\mathrm{OH}$ as the drug's affinity for $\alpha$-adrenergic receptors increases [24]. The association of $\mathrm{OH}$ with SSRI antidepressants $[8,16,25]$ and benzodiazepines $[26,27]$ has been described in previous studies, but the underlying mechanism remains unclear. Finally, antiparkinsonian drugs such as levodopa and selegiline are known to induce $\mathrm{OH}$ independently of the presence of autonomic dysfunction, probably due to vasodilation and reduced sympathetic outflow $[28,29]$.

Iatrogenic $\mathrm{OH}$ related to psychoactive medications may be of great clinical and prognostic relevance. Indeed, psychoactive drugs may also induce sedation and psychomotor slowing and negatively impact on attention, gait and balance control, thus increasing the risk of falls. Additionally, psychoactive medications are frequently prescribed in older patients at high risk of falling, as is the case of antipsychotics in older patients with cognitive impairment. In this clinical context, their hypotensive effects may further increase the risk of adverse events. Therefore, psychoactive medications should be prescribed with caution in older patients with $\mathrm{OH}$ and/or at high risk of falling.

Frail older adults may be more prone to develop iatrogenic $\mathrm{OH}$, particularly in the presence of sarcopenia. In a study by O'Connell et al., frailty was found to be associated with an abnormal BP recovery after standing [30]. Therefore, medications potentially predisposing to $\mathrm{OH}$ should be prescribed with caution in frail older people, in whom hypotension may lead to negative health outcomes such as falls, hospitalization, disability and mortality. Similarly, an intensive approach to antihypertensive treatment may lead to more harm than benefits in these patients. Indeed, evidence supporting an intensive blood pressure control derive from clinical trials involving predominantly healthy patients, with mild levels of frailty [31]. Conversely, frailer adults and those with orthostatic hypotension were excluded, which limits the transferability of these results to the "real world" geriatric population.

Given the relevant role of muscle mass in the blood pressure response to gravity stress $[32,33]$, also sarcopenia may increase the risk of iatrogenic $\mathrm{OH}$. Indeed, it may exacerbate the hypotensive effects of medications that mediate an increase in venous pooling and/or myorelaxation, such as vasodilators and benzodiazepines [34]. Conversely, ACE-inhibitors seem to positively impact on physical performance [35], which may at least partly explain their low-risk profile in the context of drug related $\mathrm{OH}$.

Given the above, a careful medication review should be carried out in patients presenting with an orthostatic blood pressure fall, with a view to minimize the risk of iatrogenic $\mathrm{OH}$. The following practical suggestions may be helpful to optimize medical therapy in these patients.

- Re-evaluate the indication of potentially hypotensive medications and consider drug withdrawal in case of inappropriate prescription

- If withdrawal is not possible, consider dosing adjustments to achieve to lowest effective dose, since hypotensive effects are usually dose-related

- Suggest a low initial dose and slow progressive up-titration according to clinical response

- Consider evening administration for medications with hypotensive and/or sedative effects, since the orthostatic blood pressure fall is usually more relevant in the morning

- For each drug class, prefer molecules showing reduced hypotensive effects, e.g. selective beta-blockers instead of mixed alpha- and beta-receptors blockers

- If available, prefer prolonged drug-release formulations, resulting in lower peak plasma concentrations

- Modify drug dosing as appropriate in case of renal impairment, to avoid accumulation.

In addition to medication review, additional risk factors for $\mathrm{OH}$ should be addressed and counselling should be provided concerning non-pharmacological treatment of $\mathrm{OH}$, including appropriate hydration, physical countermeasures, use of abdominal binders and compressive stockings, and physical conditioning.

In conclusion, pharmacological therapy is a common cause of $\mathrm{OH}$, particularly in older patients. Both cardiovascular and psychoactive medications may impact on the blood pressure response to orthostatism, thus inducing or exacerbating $\mathrm{OH}$. Therefore, a careful medication review is advisable in people presenting with $\mathrm{OH}$, with a view to minimize iatrogenic causes.

\section{References}

1. Freeman R, Wieling W, Axelrod FB, et al. Consensus statement on the definition of orthostatic hypotension, neurally mediated syncope and the postural tachycardia syndrome. Auton Neurosci 2011;161:46-8.

2. Kamaruzzaman S, Watt H, Carson C, Ebrahim S. The association between orthostatic hypotension and medication use in the British Women's Heart and Health Study. Age Ageing 2010;39:51-6. 
3. Finucane C, O'Connell MD, Fan CW, et al. Age-related normative changes in phasic orthostatic blood pressure in a large population study: findings from The Irish Longitudinal Study on Ageing (TILDA). Circulation 2014;130:1780-9.

4. Ceccofiglio A, Mussi C, Rafanelli M, et al. Increasing prevalence of orthostatic hypotension as a cause of syncope with advancing age and multimorbidity. J Am Med Dir Assoc 2019;20:586-8

5. Fedorowski A, Stavenow L, Hedblad B, et al. Orthostatic hypotension predicts all-cause mortality and coronary events in middle-aged individuals (The Malmo Preventive Project). Eur Heart J 2010;31:85-91.

6. Wolters FJ, Mattace-Raso FU, Koudstaal PJ, et al. orthostatic hypotension and the long-term risk of dementia: A populationbased study. PLoS Med 2016;13:e1002143.

7. Montastruc JL, Laborie I, Bagheri H, Senard JM. Druginduced orthostatic hypotension. A five-year experience in a regional pharmacovigilance centre in France. Clin Drug Investig 1997;14:61.

8. Poon IO, Braun U. High prevalence of orthostatic hypotension and its correlation with potentially causative medications among elderly veterans. J Clin Pharm Ther 2005;30:173-8.

9. ElDesoky ES. Pharmacokinetic-pharmacodynamic crisis in the elderly. Am J Ther 2007;14:488-98.

10. Fedorowski A, Burri P, Melander O. Orthostatic hypotension in genetically related hypertensive and normotensive individuals. J Hypertens 2009;27:976-82.

11. Fotherby MD, Iqbal P. Antihypertensive therapy and orthostatic responses in elderly hospital in-patients. J Hum Hypertens. 1997; 11:291-4.

12. Valbusa F, Labat C, Salvi P, et al. Orthostatic hypotension in very old individuals living in nursing homes: the PARTAGE study. J Hypertens 2012;30:53-60.

13. Di Stefano C, Milazzo V, Totaro S, et al. Orthostatic hypotension in a cohort of hypertensive patients referring to a hypertension clinic. J Hum Hypertens 2015;29:599-603.

14. Masuo K, Mikami H, Ogihara T, Tuck ML. Changes in frequency of orthostatic hypotension in elderly hypertensive patients under medications. Am J Hypertens 1996;9:263-8.

15. Wong AK, Lord SR, Sturnieks DL, et al. Angiotensin systemblocking medications are associated with fewer falls over 12 months in community-dwelling older people. J Am Geriatr Soc 2013;61:776-81.

16. Press Y, Punchik B, Freud T. Orthostatic hypotension and drug therapy in patients at an outpatient comprehensive geriatric assessment unit. J Hypertens 2016;34:351-8.

17. Canney M, O'Connell MD, Murphy CM, et al. Single agent antihypertensive therapy and orthostatic blood pressure behaviour in older adults using beat-to-beat measurements: The Irish longitudinal study on ageing. PLoS One 2016;11:e146156.

18. Ylitalo R, Kähönen M, Nieminen T, et al. Effects of a mononitrate, a beta1-blocker and a dihydropyridine calcium channel blocker on cardiovascular responsiveness to passive orthostasis: a placebo-controlled double-blind study in normotensive volunteers. Arzneimittelforschung 2005;55:160-6.
19. Krum H, Conway EL, Broadbear JH, et al. Postural hypotension in elderly patients given carvedilol. BMJ 1994;309:775-6.

20. Gaxatte C, Faraj E, Lathuillerie O, et al. Alcohol and psychotropic drugs: risk factors for orthostatic hypotension in elderly fallers. J Hum Hypertens 2017;31:299-304.

21. Testa G, Ceccofiglio A, Mussi C, et al. Hypotensive drugs and syncope due to orthostatic hypotension in older adults with dementia (Syncope and Dementia Study). J Am Geriatr Soc 2018;66:1532-7.

22. Brunton LL, Parker KL, Blumenthal DK, Buxton ILO. Goodman and Gilman's manual of pharmacology and therapeutics. 12th ed. New York: The Mac Graw Hill Company; 2011.

23. Mackin P. Cardiac side effects of psychiatric drugs. Hum Psychopharmacol 2008;231:S3-14.

24. Leung JY, Barr AM, Procyshyn RM, et al. Cardiovascular sideeffects of antipsychotic drugs: the role of the autonomic nervous system. Pharmacol Ther 2012;135:113-22.

25. Briggs R, Carey D, McNicholas $\mathrm{T}$, et al. The association between antidepressant use and orthostatic hypotension in older people: a matched cohort study. J Am Soc Hypertens 2018;12:597-604.e1.

26. Milazzo V, Stefano CD, Servo S, et al. Drugs and orthostatic hypotension: Evidence from literature. J Hypertens 2012;1:104.

27. Craig GM. Clinical presentation of orthostatic hypotension in the elderly. Postgrad Med J 1994;70:638-42.

28. Wood LD. Clinical review and treatment of select adverse effects of dopamine receptor agonists in Parkinson's disease. Drugs Aging 2010;27:295-310.

29. Churchyard A, Mathias CJ, Lees AJ. Selegiline-induced postural hypotension in Parkinson's disease: a longitudinal study on the effects of drug withdrawal. Mov Disord 1999;14:24651.

30. O'Connell MD, Savva GM, Finucane C, et al. Impairments in hemodynamic responses to orthostasis associated with frailty: Results from the Irish Longitudinal Study on Ageing (TILDA). J Am Geriatr Soc 2018;66:1475-83.

31. Russo G, Liguori I, Aran L, et al. Impact of SPRINT results on hypertension guidelines: implications for "frail" elderly patients. J Hum Hypertens 2018;32:633-8.

32. Wieling W, Krediet CT, van Dijk N, et al. Initial orthostatic hypotension: review of a forgotten condition. Clin Sci (Lond) 2007;112157-65.

33. Krediet CT, Go-Schön IK, Kim YS, et al. Management of initial orthostatic hypotension: lower body muscle tensing attenuates the transient arterial blood pressure decrease upon standing from squatting. Clin Sci (Lond) 2007;113:401-7.

34. Rivasi G, Kenny RA, Ungar A, et al. Effects of benzodiazepines on orthostatic blood pressure in older people. Eur J Intern Med 2020;72:73-8.

35. Liguori I, Russo G, Aran L, et al. Sarcopenia: assessment of disease burden and strategies to improve outcomes. Clin Interv Aging 2018;13:913-27. 